\title{
Categorical perception of color: evidence from secondary category boundary
}

\author{
This article was published in the following Dove Press journal: \\ Psychology Research and Behavior Management \\ 25 November 2015 \\ Number of times this article has been viewed
}

\author{
Abdulrahman Saud \\ Al-rasheed \\ Department of Psychology, \\ King Saud University, Riyadh, \\ Kingdom of Saudi Arabia
}

Correspondence: Abdulrahman Saud Al-rasheed

Department of Psychology,

King Saud University, Riyadh 12372 ,

Kingdom of Saudi Arabia

Tel +966 II 4674796

Email asalrasheed@ksu.edu.sa
Abstract: Despite a plethora of behavioral research exploring the phenomenon of color categorical perception (CP) known as "better discrimination between pair of colors stimuli from different categories and pair of colors stimuli from the same category even when the stimulus differences between the pairs of stimuli are equal", most of the evidence for the CP of color was derived from Roman or top-to-down script readers and very rarely from right-to-left script readers in primary category. To date, no studies of color CP have been conducted on right-to-left script readers in secondary category boundary to support this theory. Three experiments have been conducted: Experiments 1 and 2 established the Arabic blue-purple secondary category boundary, and Experiment 3 tested the $\mathrm{CP}$ of color in the blue-purple category boundary. Sixty participants (30 men and 30 women) took part in this study. All spoke Arabic as their first language, and all were undergraduate or postgraduate students at King Saud University. Their ages ranged from $18-35$ years with a mean age of 21.9 years $(\mathrm{SD}=5.2)$. The result indicated that for Experiments 1 and 2, it appeared that the Arabic blue-purple category boundary was approximately 10PB and it is in the same location as for English. For Experiment 3, reaction times in the between-categories condition were significantly faster than those in the withincategory condition; this suggested that $\mathrm{CP}$ of color was shown in the Arabic's blue-purple secondary category boundary.

Keywords: categorical perception, CP of color, categorization, blue-purple category boundary, secondary category boundary

\section{Introduction}

Although all humans with normal trichromatic color vision have the same general physiological basis of color vision, ${ }^{1}$ there is noticeable diversity among languages in the way they categorize the continuum of visible colors. Some languages are reported to use as few as two terms to describe all colors ${ }^{2}$; others use many more. ${ }^{3,4}$ Although a considerable amount of material has been written on this subject, relatively little has been written on color categorization in Arabic.

The logic of the experiments presented in this study, on color $\mathrm{CP}$, requires that the differences between chosen pairs of colors are equal according to some metric. The metrics most commonly used in the literature are derived from either the Munsell system $^{5}$ or Commission Internationale de 1'Eclairage (International Commission on Illumination) (CIE) ${ }^{6}$ systems (Supplementary materials). Both aim to represent colors in "perceptually uniform" color space. That is, distances within each spatial coordinate system are intended to represent "perceptual distance": the perceived dissimilarity between colors. The greater the spatial separation between two colors is, the greater the perceived dissimilarity between the two colors should be. 
The universal color category hypothesis that all languages draw their basic color terms from a universal inventory of just eleven color categories was examined in a series of experiments on Arabic language speakers. ${ }^{7-9}$ It was found that Arabic speakers have eleven basic color categories, like English, that correspond to Berlin and Kay's ${ }^{10}$ universal categories. More importantly, as Arabic proved to have eleven basic categories, a further study of color CP in Arabic speakers has been investigated in the blue-purple primary category boundary to shed light on the role of language in $\mathrm{CP} .{ }^{11}$

Current study examines a color category effect (categorical perception $[\mathrm{CP}]$ of color) whereby the category of colors appears to affect color discrimination. Previous research has found color category effect in the Arabic blue-purple primary category boundary. ${ }^{11}$ Here, the author investigates this color category effect, by considering whether it will be shown in a secondary color region such as blue-purple region. This was the prime reason for the current study.

\section{Historical and conceptual framework}

The origin and nature of color and our perception of color have intrigued philosophers and scientists, from at least the time of Aristotle ( $\sim 350 \mathrm{BC})$. Although the ontology of color continues to be debated by philosophers, ${ }^{12}$ there is a consensus among scientists that color perception derives from an interaction between the physical properties of light (wavelength and intensity) and the way our visual system responds to the physical stimulus. Although this statement might seem tautological, it has an important implication, recognized by two great physicists, that color is, in part, a matter of psychology. "For the Rays to speak properly are not coloured. In them there is nothing else but a certain Power and Disposition to stir up a Sensation of this or that Colour." ${ }^{13}$ And, similarly, "Now if the sensation which we call colour has any laws, it must be something in our own nature which determines the form of these laws." ${ }^{14}$ This recognition of the roles of the visual system led directly to posing the general questions mentioned above. What might be the role of the visual system in influencing color language? If there were such influences, were they the same for everybody - Universalism - or might individual visual systems differ leading to differences in color language - Relativism? Moreover, if visual systems differed, did the differences arise from experience - Nurture - or were they inherited - Nature?

\section{Color language}

Gladstone ${ }^{15}$ was probably the first to draw attention to how languages differed in the ways they named colors. He noted that the way Homer described colors differed markedly from the way Victorian English described color. Moreover, he attributed this difference to differences in color perception: "that the organ of colour and its impressions were but partially developed among the Greeks of the heroic age" (pp 457-499). Meanwhile, Geiger ${ }^{16}$ extended Gladstone's survey of ancient literature and concurred with Gladstone that the ancients used fewer color terms than found in English, which he too attributed to differences in color vision. He suggested that color term systems "evolved" systematically acquiring new terms reflecting the parallel evolution of color vision.

Rivers ${ }^{17}$ seemed to have found the first empirical evidence linking differences in color language to differences in color vision. On finding that the languages of the Torres Straits had fewer color terms than English, and that they did not distinguish blue from purple, he attributed these differences to differences in color vision.

Of course, it should be noted straight away that in all three cases above, what had been observed was an association between (presumed) color vision and color language; even granted the validity of the observation, the attribution of "perception-causing language" does not necessarily follow. Language could change color perception, consistent with the yet-to-be formalized Linguistic Relativity Hypothesis (LRH). ${ }^{18}$

Even as early as 1877 , Magnus, ${ }^{19}$ using recently collected data, confirmed that languages differed in the way Geiger had noted but failed to find the corresponding differences in color vision. In the early days of the development of scientific psychology, Woodworth, ${ }^{20}$ while criticizing Rivers, offered an explanation of Magnus's data: differences between languages reflected differences in cultural needs - the Utilitarian Hypothesis. This hypothesis came to dominate American Linguistics and Anthropology for the next 60 years. For example, Ray's views were typical: "Each culture has taken the spectral continuum and divided it into units on a quite arbitrary basis." ${ }^{21}$

Many of the issues summarized above are still debated today. Berlin and Kay ${ }^{10}$ offered an updated version of the Geiger/Magnus evolutionary hypothesis, and subsequently argued that the evolutionary path was constrained by universal visual physiology. ${ }^{22}$ Although a considerable amount of data supports their position, both the data and the interpretation are disputed. ${ }^{23,24}$

\section{Color language and color perception}

As mentioned above, as early as the late 19th century, Gladstone, Geiger, and subsequently, Rivers all assumed that 
differences in color language reflected differences in color perception. This was consistent with the commonsense view that language was determined by thought, where thought in its widest sense included perception. Language was taken to be the "servant" of thinking being "merely" a way of expressing thoughts. This view was turned on its head most prominently by Whorf ${ }^{18}$ who argued that rather than being a passive way of expressing thoughts, language determined (heavily constrained) thought: "We dissect nature along lines laid down by our language.’

This LRH was first introduced to experimental psychologists by Brown and Lenneberg. ${ }^{25}$ They investigated the relationship between recognition memory for colors and the "codability" or ease of naming of the colors. They found that the easier it was to describe a color to someone else so that they could recognize the color among distractors, the easier it was to remember (recognize) the color among distractors. They argued that this showed language influencing memory, an aspect of thought. This implies that if people differed in their color language, so should their memory for particular colors.

Heider, in a series of classic studies, ${ }^{2,26}$ tested this implication by comparing the Dani of Iranjia whose language had only two color terms, with English-speaking Americans. In one task, he compared recognition memory for good examples (foci) of English chromatic terms, with memory for less prominent examples. As for English speakers, names for foci were more salient than names for less good examples; following Brown and Lenneberg, memory for focals should be better than for nonfocals. And, indeed, this was the result. If this pattern results from language influencing memory, then it should not be found for the Dani, who had no distinctive names for the colors. However, the Dani showed the same pattern as the English speakers. Rosch ${ }^{27}$ attributed the results to the greater perceptual salience of the focals than of the nonfocals, and she attributed this to the way the visual system processed color, and she assumed that this was in common to all people.

Curiously, for a study just comparing two languages, and just investigating one domain (color), Rosch's results were taken as showing that color perception was universal, and that language did not influence perception. Despite this small empirical base, the Zeitgeist shifted from the presumption of Relativity to the presumption of Universalism, and there was very little further empirical work until interest in the issue was rekindled by, among others, Davies and Corbett ${ }^{28}$ and Roberson et al. ${ }^{24}$ This new wave of interest was centered on the phenomenon of $\mathrm{CP}$ - better discrimination between colors from different categories than colors from the same category. ${ }^{29}$ As colors from different categories also have different names, the question arose of whether $\mathrm{CP}$ was due to the use of language in some way, and if it were, this would be evidence for the LRH.

\section{Physiology of color vision}

Color vision is usually defined as the ability to discriminate stimuli of equal brightness that differ in their spectral composition. In humans, this ability is associated with our experience of color, although there appears to be no necessary connection between the two. The causal chain leading to perceptual experience starts with the nature of light, continues with the interaction between surfaces in the world and light, then the formation of the retinal image, followed by recovery of information about the relative spectral distribution of light at each point on the retina. A great deal is now known about this casual chain involving physics, molecular biology, genetics, and neurology leading Mollon to claim that color perception is the first case of understanding the pathway from physics through to conscious experience ${ }^{30}$ (see Ref 30 for reviews). The aim here, however, is to provide the basis for understanding Kay and McDaniel's appeal to perceptual physiology to explain the origin and nature of $\mathrm{CP}$ of color. In this case, what is needed is an explanation of the "cardinal directions of color space", 33 and this is given below.

Sunlight, and most artificial substitutes, is composed of a mixture of different wavelengths including those in the range of approximately $400-700 \mathrm{~nm}$, in approximately equal amounts. We experience such spectral mixtures as white or gray (achromatic). However, surfaces in the world change this equal-energy composition. The chemical structure of the surface material selectively absorbs some wavelengths and reflects the remainder. For instance, a surface appears red if it reflects long wavelengths more strongly than shorter wavelengths. The structure of light incident on an eye in the world "preserves" its history of reflections. The optics of the eye preserves the spatial history of its trajectory: light reflected from adjacent points on a surface falls on adjacent points on the retina of the eye, hence forming an "image" of the world. Thus, the boundary between two objects (an edge) also forms a boundary in the image. If the spectral composition of the light reflected from either side of the edge differs, then if the visual system could detect differences in wavelength, it would have the potential to detect the edge. Thus, sensitivity to wavelength has the potential to allow recovery of the layout of objects in the world. 
The visual system has evolved to exploit the properties of visual stimulation described above. The key first stage is that the retina contains four different kinds of light-sensitive receptors, the rods and three kinds of cones. Each kind of receptor, although sensitive to a broad range of wavelengths, responds more strongly to some wavelengths than others. Moreover, the distribution of spectral sensitivities varies across types of receptors, due to differences in the "photopiment" - the lightsensitive chemical - they contain. As the rods are only operative at low illumination levels, in normal daylight, the primary information for vision is carried by the relative signal strengths of the three kinds of cone. The cones are usually designated as long (L), medium (M), and short (S) reflecting their relative peak sensitivities $(560 \mathrm{~nm}, 530 \mathrm{~nm}$, and $420 \mathrm{~nm})$. $^{31,32}$

The trichromatic stage at the receptor level is transformed into three opponent channels in the ganglion cells of the retina. Different combinations of the $\mathrm{L}$ and $\mathrm{M}$ cones are involved in each channel, but the $\mathrm{S}$ cone is primarily involved in just one channel. The luminance channel signals in proportion to the sum of the $\mathrm{L}$ and $\mathrm{M}$ cones $(\mathrm{L}+\mathrm{M})$. The other two channels carry potential information about relative wavelength. One encodes the difference between $\mathrm{L}$ and $\mathrm{M}$ signal strengths $(\mathrm{L}-\mathrm{M})$, while the other signals the difference between the sum of $\mathrm{L}$ and $\mathrm{M}$, and the $\mathrm{S}$ cone signal strength ([L-M]-S). As well as the functional separation of these channels, they are also anatomically separate, being carried by distinct kinds of ganglion cells, and the three pathways (magno, parvo, and konio) following different pathways from retina to lateral geniculate nucleous (LGN), to V1.

When opponent process neurons were first discovered in monkey LGN, ${ }^{34}$ it was thought that they were the neurological substrate of Hering's conjectured opponent primaries. ${ }^{35}$ Hering suggested that there were four "unique hues", red, purple, yellow, and blue, and that all other colors appeared to be mixtures of these primary experiences. Orange, for instance, was a blend of red and yellow, and purple was a blend of red and blue. Moreover, while we could experience blends such as these, there were other "impossible blends". These were red-purple and blue-yellow, and Hering thought that his four primaries were organized into these mutually antagonistic pairs. De Valois and Jacobs originally thought that the peak activity of their newly discovered opponent process neurons was red or purple and blue or yellow, but it later became clear that their primary axes were more like magenta-chartreuse and orange-turquoise. ${ }^{34}$ Nevertheless, the activity of these opponent process neurons has been taken to signal the cardinal directions of color space, and to underlie color perception.

\section{Color-order systems}

The logic of the experiments presented in this study, on color $\mathrm{CP}$, requires that the differences between chosen pairs of colors are equal according to some metric. The metrics most commonly used in the literature are derived from either the Munsell system or the CIE systems (Supplementary materials). Both aim to represent colors in "perceptually uniform" color space. That is, distances within each spatial coordinate system are intended to represent "perceptual distance": the perceived dissimilarity between colors. The greater the spatial separation between two colors is, the greater the perceived dissimilarity between the two colors should be.

Although both systems attempt to represent perceptual distance, they were derived by quite different methods. The Munsell system was empirically derived, and in its current form, is based on 40 observers making similarity/difference judgments between many color pairs; the total number of judgments runs into the tens of millions..$^{5}$ In contrast, the CIE system is modeled on the relative spectral sensitivities of the three types of cone (L, M, and S; "Aim of the study") in the "standard CIE observer". The CIE coordinate system is thus three dimensional, based on three "primaries", but for reasons of convenience, the cone primaries have been transformed into the CIE primaries $X, Y$, and $Z$. One advantage of using these primaries is that all perceivable colors can be represented in a simple chromaticity diagram whose axes are $x$ and $y$, where $x=X /(X+Y+Z)$ and $y=(Y /(X+Y+Z)$. As $x$ and $y$ are proportional values, by implication, there is a third coordinate, $z(z=Z /(X+Y+Z)$, which is not explicitly represented in the diagram. For present purposes, the key point is that a further transformation from $x$ and $y$ to $u^{\prime}$ and $v^{\prime}$ results in a diagram where to a first approximation, distance in the diagram represents perceptual distance. Moreover, a further transformation to $L^{*} u^{*} v^{*}$ yields a color "space" where Euclidean distance $(\Delta E)$ provides a quantitative measure of perceptual distance.

Both Munsell and Commission Internationale de l'Eclairage (CIELUV) try to provide a measure of perceptual distance on at least an interval scale, and if they have succeeded, the two distance measures should correspond. The two measures agree to a good first approximation, but the correspondence is not perfect.

\section{The current study}

The color spectrum is a physical continuum, but it is perceived discontinuously, as discrete categories or segments of hues. ${ }^{37}$ This is part of an effect called CP. CP is found when a continuum is divided into categories, and when these categories 
appear to affect discrimination. In operational terms, CP can be defined by faster and/or more accurate discrimination of pair of stimuli that cross a category boundary (across category), than two stimuli from the same category (within category), even when the stimulus differences between the pairs of stimuli are equal. This definition of $\mathrm{CP}$ will be used throughout, and is illustrated in the classic form shown in Figure 1.

Figure 1 shows three stimuli designated as $7.5 \mathrm{~PB}, 2.5 \mathrm{~PB}$, and 7.5PB. One stimulus (7.5PB) belongs to the linguistic category blue, and two (2.5P, 7.5P) belong to the linguistic category purple, with the category boundary between $7.5 \mathrm{~PB}$ and 2.5P. The separation between the stimuli is equal. Discrimination of the cross-category stimulus pair (7.5PB, $2.5 \mathrm{P}$ ) is faster and/or more accurate than discrimination of the within-category stimuli (2.5P, $7.5 \mathrm{P})$.

$\mathrm{CP}$ has been reported on a wide range of color perception tasks. For example, recognition memory and $\mathrm{X}-\mathrm{AB}$ tasks, ${ }^{24,38-41}$ same-different tasks, ${ }^{29,42}$ similarity judgments, ${ }^{39,43}$ and target detection and visual search tasks. ${ }^{44}{ }^{46}$ In the 2-X$\mathrm{AB}$ task, a target stimulus (eg, blue1) is presented followed by two test stimuli; one of the test stimuli is identical to the target, and the other one (the foil) is different. The foil can be either from same category as the target (eg, blue2) or from a different category (eg, green1). The task is to decide as fast as possible which of the test stimuli is identical to the target. The results showed that target identification was faster and/or more accurate for different category than same-category foils. In the search task, a target stimulus is presented among other stimuli (distractors); the distractors can either be from the same category as the target (eg, blue1 among blue2s) or from a different category to the target (blue1 among purple1s). The task is to detect the location of the target as fast as possible. Detection of a target that is from a different category to the distractors is faster and/or more accurate than detection of a target from the same category as the distractors.

Although CP has been reported in a wide range of studies, it is not clear what the origin and nature of this effect are. The degree to which language and perception contribute to the

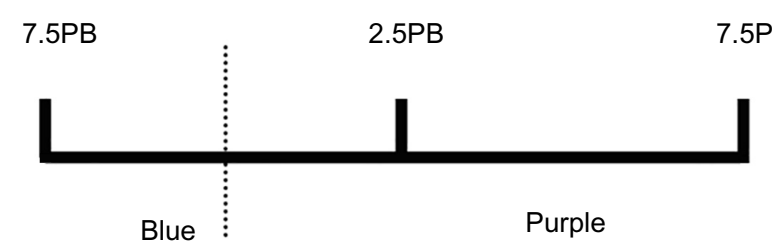

Figure I Diagram representing categorical perception.

Notes: Munsell codes of the stimuli; stimuli varied in hue at constant value and chroma. Hue separations were 5 Munsell hue units apart. The dashed line indicates the category boundary. category effect has been extensively debated. To investigate the contribution of language to $\mathrm{CP}$, recent studies have considered how the effect is lateralized. ${ }^{47-49}$ And, they reasoned that, as the left hemisphere ( $\mathrm{LH})$ is dominant for most language functions, if color $\mathrm{CP}$ is related to language, it should be stronger in the LH. To test this, Gilbert et al ${ }^{47}$ used a visual search task where targets were lateralized to the left or right visual field (RVF). Stimuli were shown in a display of 12 colored squares in a clock shape; eleven of the squares (the distractors) were identical in color, and one (the target) was different. The relationship between the distractors and the target stimulus was manipulated, so targets and distractors were either from the same color category or from a different color category. While looking at a central fixation cross, participants had to decide whether the target was to the left or to the right of fixation. They found that response times (RTs) were faster when target and distractors were different categorically than when target and distractors were just perceptually different. However, this category effect was found larger when the target was presented to the RVF. They argued that this pattern of lateralization was consistent with $\mathrm{CP}$ being due to the implicit use of language.

The $\mathrm{LH}$ bias in color $\mathrm{CP}$ has been related to the linguistic nature of the $\mathrm{LH}$, and converging evidence to support this hypothesis has been reported on a wide range of color perception tasks and methods, for example, functional magnetic resonance imaging, ${ }^{50}$ the event-related potential technique, ${ }^{51}$ and grid search task..$^{52}$ It is also possible that other factors contribute the LH bias in category effect in adults as the influence of reading habits to perception has been shown in several studies. ${ }^{53-58}$

Although it is not entirely clear how to predict the effect of habitual reading direction on CP of color, the author decided that it was worth exploring to test the generality of categorical effects of color across cultures and reading directions. Han and Northoff ${ }^{59}$ describe several instances of cultural differences being associated with differing neural organizations, and argue that in general, it is a good practice to test the generality of findings across a range of cultures. Their exhortation is consonant with the motivation behind this study, which is to test that patterns of $\mathrm{CP}$ of color are generalizing across cultures and reading directions.

\section{Aim of the study}

Despite a plethora of behavioral research exploring the origin and nature of color $\mathrm{CP}$, most of the evidence for the $\mathrm{CP}$ of color was derived from Roman script readers in primary and secondary boundaries and as, to date, no studies of color CP 
have been conducted on Arabic in secondary boundary (bluepurple) to support of this theory. The main aim of this study is to investigate whether the color CP can be shown in participants whose reading direction is from right to left as in Arabic in (blue-purple) secondary color category boundary.

A preliminary study by Al-rasheed ${ }^{11}$ established the location of the azrock "blue"-akhdar "green" boundary in Arabic. As the boundary was at more or less the same location as in English (7.5BG), then CP of color was tested in a visual search task, and a target detection task for the azrock "blue"-akhdar "green" boundary. However, there have been no studies that have established the Arabic blue-purple secondary category boundary. The other goal of this study is to identify the Arabic blue-purple category boundary. The purposes of this research and the procedures that were undertaken were explained to the participants. All participants' questions were fully answered. All participants understood the explanations and gave informed consent. This study was ethically approved by the review board.

\section{Experiment I: lightness and saturation for the blue-purple category boundary Introduction}

The overall aim of this study was to establish the Arabic blue-purple category boundary. Experiment 1 determined the saturation and lightness levels in which stimuli were named purple and blue with high agreement. Experiment 2 then estimated where the boundary was using hues at the value $(\mathrm{V}) /$ chroma $(C)$ combination found in Experiment 1.

\section{Method}

\section{Participants}

Twenty participants, ten male and ten female, took part in the value and chroma task. They spoke Arabic as their first language, and all were students at King Saud University. Based on self-reports, all were right-handed and had normal color vision, as indicated by the City University Test. ${ }^{60}$ Their ages ranged from 18 years to 25 years with a mean age of 21.05 years $(\mathrm{SD}=2.79)$.

\section{Stimuli}

Thirty-six stimuli were used made up from the combination of six hues (2.5PB, 5PB, 7.5PB, 10PB, 2.5P, and 5P)

\begin{tabular}{|c|c|c|c|c|c|c|}
\hline Blue & 2.5PB & $5 \mathrm{~PB}$ & 7.5PB & 10PB & $2.5 \mathrm{P}$ & $5 P$ \\
\hline
\end{tabular}

Figure 2 Munsell codes of the stimuli used in Experiment I.

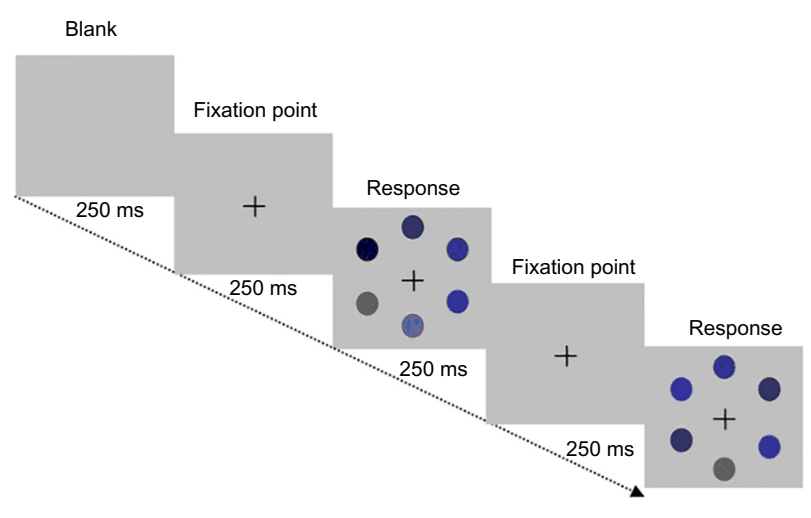

Figure 3 Example of the hue selection task.

Notes: Each display contained the same hue (circles) at each of six different combinations of value and chroma. The six hues were used in different displays.

(Figure 2) at each of six combinations of value and chroma $(4 / 10,5 / 10,6 / 10,5 / 9,6 / 8$, and 7/10). Each hue set was presented as six circular stimuli (diameter $=5.5 \mathrm{~cm}$; visual angle $=6.5^{\circ}$ ) equally spaced on the circumference of an imaginary circle around a fixation cross in the center of the screen, on a neutral gray background (Figure 3). $\operatorname{The}^{\mathrm{CIE}} \mathrm{Y}, x$, and $y$ chromaticity coordinates of the gray point of the monitor were $19.47 \mathrm{~cd} / \mathrm{m}^{2}, 0.336 \mathrm{~cd} / \mathrm{m}^{2}$, and $0.344 \mathrm{~cd} / \mathrm{m}^{2}$, respectively.

\section{Equipment}

Stimuli were displayed on a 17 -inch calibrated CRT Sony Trinitron monitor (model GDM-F520). Color readings were made using a Cambridge Research Systems ColorCAL colorimeter (Rochester, UK). An example of the task is given in Figure 3.

\section{Procedure}

The experiment was conducted in a dark room. On each trial, one hue set was presented and remained on the screen until a response was made. Participants viewed the display at a distance of $60 \mathrm{~cm}$. They were told that on each trial, the display would consist of six colored stimuli in a clock shape against a gray background. Their task was to decide which one of these five stimuli was the best example of either purple or blue. Responses were made verbally and recorded by the experimenter.

\section{Results and discussion}

The percentage of participants selecting each color as the best example, for each hue, is shown in Table 1 .

As can be seen, 5/10 was the most frequently selected "best example" for the four hues 5PB, 7.5PB, 10PB, and 2.5P with a score of approximately $80 \%$ and for the last hue $5 \mathrm{P}$ with a score of $70 \%$. However, stimulus $4 / 10$ was the best 
Table I The percentage of times that each stimulus was chosen as the best example of either blue or purple Arabic terms

\begin{tabular}{lllllll}
\hline VIC & 2.5PB & 5PB & 7.5PB & I0PB & 2.5P & 5P \\
\hline $4 / 10$ & 80 & 0 & 0 & 0 & 0 & 0 \\
$5 / 10$ & 0 & 60 & 80 & 80 & 80 & 70 \\
$6 / 10$ & 0 & 20 & 20 & 20 & 20 & 30 \\
$5 / 9$ & 20 & 10 & 0 & 0 & 0 & 0 \\
$6 / 8$ & 0 & 10 & 0 & 0 & 0 & 0 \\
$7 / 10$ & 0 & 0 & 0 & 0 & 0 & 0
\end{tabular}

Abbreviation: $V / C$, value/chroma.

example for $2.5 \mathrm{~PB}$ with a score of $80 \%$, and stimulus $6 / 10$ was the second most frequently chosen for the hues $5 \mathrm{~PB}$, 7.5PB, 10PB, 2.5P, and 5P.

In summary, Experiment 1 was designed to identify the value and chroma for which the hues are defined as blue or purple in Arabic. Arabic participants showed that 5/10 had high percentage agreement for hues $5 \mathrm{~PB}, 7.5 \mathrm{~PB}, 10 \mathrm{P}$, and $2.5 \mathrm{~PB}$ in the blue-purple category and $4 / 10$ value and chroma was the highest agreement only for $2.5 \mathrm{~PB}$.

\section{Experiment 2: color naming for the blue-purple region Introduction}

Experiment 1 showed that stimuli were the best example at value 5 and chroma 10 for the six hues tested. The aim of the current experiment was to identify location of the hue boundary for the Arabic blue-purple category boundary before conducting research on the blue-purple color $\mathrm{CP}$ in Arabic in the next experiment.

\section{Method}

\section{Participants}

Thirty participants took part in the naming task, half male and half female. Their ages ranged from 19 years to 35 years with a mean age of 22.6 years $(S D=4)$, and all were undergraduate and postgraduate students at King Saud University. Based on self-reports, all were righthanded and had normal color vision, as indicated by the City University Test. ${ }^{60}$

\section{Equipment and stimuli}

There were six stimuli used in this experiment 2.5PB, 5PB, 7.5PB, 10PB, 2.5P, and 5P. These stimuli varied only in Munsell hue with value and chroma kept constant on 5/10. Their CIELUV coordinates $\left(u^{*}, v^{*}\right)$ were as follows: -31.21 , $63.17 ;-23.87,-64.34 ;-11.32,-63.63 ;-0.18,-61,09$; $9.49,-56.44$; and $18.04,-50.89 ; L^{*}=51.58$. The stimuli were displayed on the same monitor that was used in
Experiment 1 and were measured with the ColorCAL colorimeter used in the previous experiment.

\section{Procedure}

A rectangular shape $(120 \mathrm{~mm} \times 60 \mathrm{~mm})$ on a gray background $(40 \mathrm{~cm} \times 30 \mathrm{~cm})$ was presented on a monitor in a darkened room at a viewing distance of $60 \mathrm{~cm}$. Stimuli were viewed one at a time, in a random order, remaining on display until a naming response was made. Responses were made using a computer keyboard. There were five repetitions of each stimulus, and the 30 trials were in a random order. The task was to label the stimuli as banafsagee "purple", azrock "blue" or azrock-banafsagee "blue-purple" if the participant could not decide whether the stimulus was purple or blue. The term azrock-banafsagee "blue-purple" was described to the participants as a color which mixed half blue and half purple (50\% purple and 50\% blue). An example of the task is shown in Figure 4.

\section{Results and discussion}

The agreement curve for blue-purple color naming for Arabic speakers is shown in Figure 5. Participants used three terms to name the six stimuli. The graph represents the percentage of blue, purple, and blue-purple responses to each of the five stimuli in the continuum.

As can be seen, for the azrock "blue" term, the agreement curve peaks on a hue value of $2.5 \mathrm{~PB}$ with $100 \%$ of the sample and gradually fell toward the purple region. Three stimuli out of six in the continuum were named azrock "blue" by over $75 \%$ of the sample (2.5PB, 5PB, and $7.5 \mathrm{~PB})$. In contrast, the agreement curve for the banafsagee "purple" term shifted toward a hue value of $5 \mathrm{P}$ with $95 \%$ of the sample and gradually fell toward the azrock "blue" region reaching the lowest point at $7.5 \mathrm{~PB}$ with $5 \%$ of the sample. Two stimuli out of six

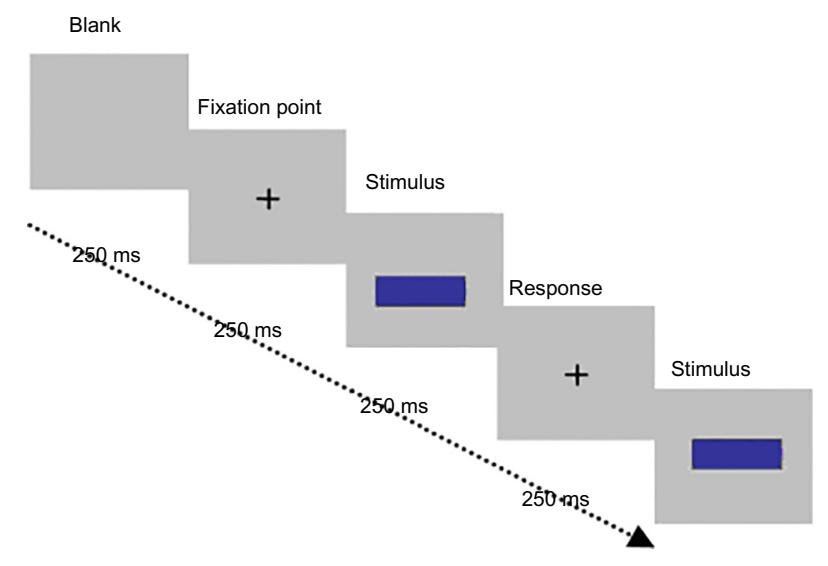

Figure 4 Example of the color naming task. Note: + Indicates the fixation point; each blue rectangle indicates the stimulus. 


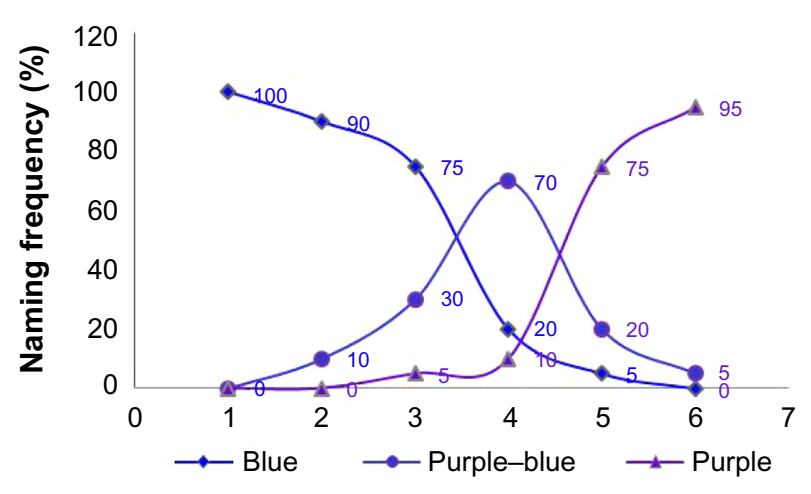

Figure 5 Percentage naming frequencies across observers for the six hues. Note: I: “2.5PB”, 2: “5PB”, 3: “7.5PB”, 4: “IOPB”, 5: “2.5P”, and 6: “5P”.

in the continuum were named banafsagee "purple" by over $75 \%$ of the sample. It can be seen that the level of consensus for the azrock-banafsagee "blue-purple" term was highest only for the stimulus 10PB when it was offered by at least $70 \%$ of the sample.

Based on these results, it appears that the Arabic azrockbanafsagee "blue-purple" category boundary was approximately $10 \mathrm{~PB}$ with the most frequent responses to the two stimuli to the left being azrock "blue" and for the two to the right of the boundary being banafsagee "purple". It appears that the Arabic blue-purple category boundary is in the same location as for English. ${ }^{61}$

\section{Experiment 3: CP of color in the blue-purple category boundary Introduction}

Previous studies investigated the color CP in Arabic blue-purple region, ${ }^{11}$ and the results showed that discrimination of pairs of color from different lexical categories (blue and purple) was faster than pairs from the same lexical category (different shades of blue or purple), even though the separation of within- and between-category pairs was equal. In addition and for the same purposes, Al-rasheed et $\mathrm{al}^{52}$ showed that $\mathrm{CP}$ of color was independent of habitual reading direction. Most tests of $\mathrm{CP}$ of color have used one or other of these tasks: $\mathrm{X}$-AB tasks, ${ }^{24,38-41}$ same-different tasks, ${ }^{29,42}$ similarity judgments, ${ }^{39,43}$ and target detection and visual search tasks. ${ }^{44-46}$ In the next experiment (Experiment 3), the grid search task was used to test the CP of color, and whether it will be shown in the blue-purple secondary category boundary.

\section{Method}

\section{Participants}

Twenty native Arabic-speaking undergraduates from the University of King Saud participated in this experiment.
There were ten males, with a mean age of 21.0 years $(\mathrm{SD}=3.09)$, and ten females, with a mean age of 21.1 years $(\mathrm{SD}=2.60)$. Their ages ranged from 18 years to 25 years. Based on self-report, all were right-handed and had normal color vision as indicated by the City University Test. ${ }^{60}$ Most of the participants participated for course credit, and a few volunteered.

\section{Stimuli and apparatus}

As shown in Figure 6, three color stimuli were used in this experiment; two blues (2.5PB and 7.5PB) and one purple (2.5P); value and chroma were kept constant $(5 / 10)$. The separation between adjacent stimuli was five Munsell hue steps (AE 15). Their CIELUV coordinates $\left(u^{*}, v^{*}\right)$ were as follows: $-31.21,63.17$; $-11.32,-63.63$; and 9.49, -56.44; $L^{*}=51.58$; a Cambridge Research Systems ColorCAL colorimeter was used to measure the CIE co-coordinates, and they were displayed on a 17-inch CRT model GDM-F520.

\section{Procedure}

Adjacent stimuli were paired, to form one within-category pair (blue1-blue2) and one between-category pair (blue1-purple1). For each pair, one stimulus was the target, and the other stimulus was used for the distractors, with both stimuli in a pair appearing equally often as distractors. The target for all trials was always blue, and the distractors were randomly switched between "within" (blue) and "across" (purple). There were equal numbers of trials for each combination of within category or between category, and the order of trials was randomized across these two categories. In addition, target location was randomized across trials with the constraint that the target appeared equally often to the left, right, top, and bottom of fixation. Stimuli were shown as $2.5 \mathrm{~cm}$ squares with $5 \mathrm{~mm}$ gaps between adjacent locations, appearing in locations specified by a $3 \times 3$ square grid on the display

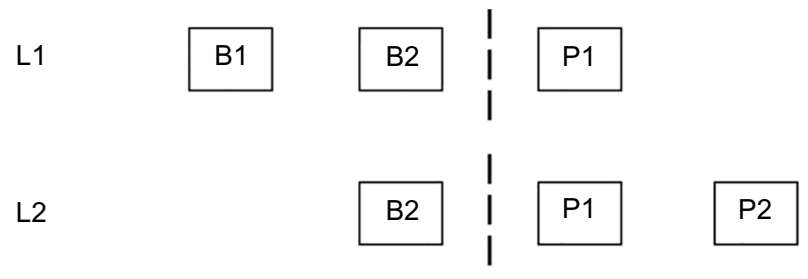

Figure 6 Illustration of the four stimuli used.

Notes: $\mathrm{LI}$ is the location of hue for the blue target (stimuli BI and B2 are from the same category, "blue", while stimulus PI belongs to a different category, "purple"). L2 is the location of hue for the purple target (stimuli P2 and P2 are from the same category, "purple", while stimulus B2 belongs to a different category, "blue"). The dashed line indicates the Arabic blue-purple boundary. 


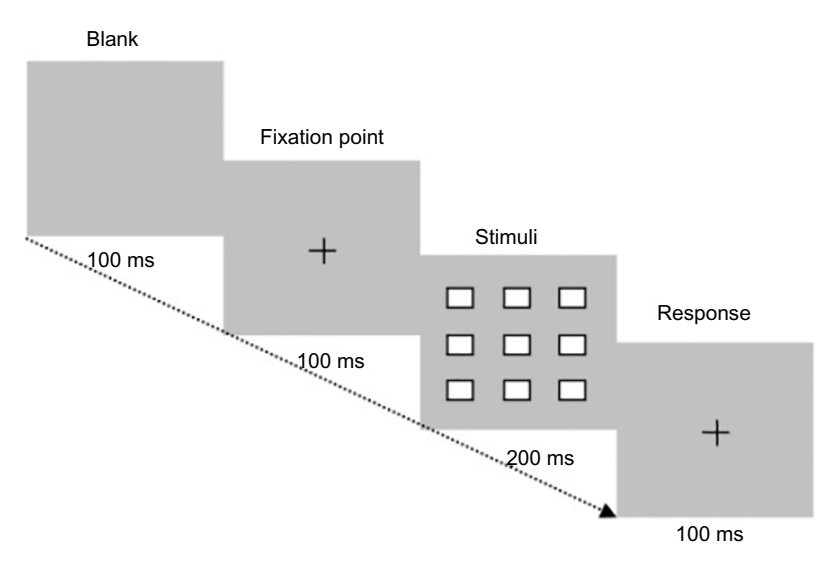

Figure 7 Example of the grid task.

Note: The white squares indicate the positions of the target and distractors; + indicates the fixation points

(Figure 7). The target appeared among eight distractors on a gray background $\left(19.47 \mathrm{~cd} / \mathrm{m}^{2}, 0.336 \mathrm{~cd} / \mathrm{m}^{2}, 0.344 \mathrm{~cd} / \mathrm{m}^{2}\right)$. The distractors plus the target stimulus locations within the grid were randomly selected.

The experiment began with a fixation cross which remained for $100 \mathrm{~ms}$ to alert the participants that the trial was beginning. Then, the test display followed and remained on screen for $200 \mathrm{~ms}$. The next trial began when the participants had responded. There were 180 trials, 90 for each category (within and cross) and 20 trials for each position. Participants were given 12 practice trials before starting the experiment, and they took approximately 10 minutes to complete the task.

The participants were tested individually in a dark room and sat with their head position constrained by a chin-rest, so that eye level was at the center of the monitor, with a viewing distance of $60 \mathrm{~cm}$. Participants were informed that they would be presented with a target stimulus among a varied number of distractors and their task was to decide whether they detect the target which will be in a different hue than the distractors. Responses were made by clicking the mouse button as soon as they see the target.

The percentage of incorrect trials was calculated for each subject, for each combination of sex (male/female) and category (cross/within). A two-way repeated measures analysis of variance on the error rates showed that the only significant effect was for category sex $(F<1)$. Cross-category error rates (mean $=2.38 \%, \mathrm{SD}=3.21$ ) were approximately $1 \%$ lower than within-category responses (mean $=3.92$, SD $=4.84), F(1,9)=0.27, \mathrm{MSE}=30.55, P<0.05$.

Median RTs for each subject were calculated for each combination of sex and category for correct trials. Although female participants (mean $=406 \mathrm{~ms}, \mathrm{SD}=54$ ) responded $\sim 31 \mathrm{~ms}$ faster than male participants (mean $=437, \mathrm{SD}=65$ ), this difference was not significant $(F(1,9)=4.93, \mathrm{MSE}=9954.0, P=0.58)$. For the category factor, there was a significant effect; cross-category responses ( mean $=380.8, \mathrm{SD}=38.1$ ) were approximately $82 \mathrm{~ms}$ faster than within-category responses (mean $=462.8$, $\mathrm{SD}=52.1 ; F(1,9)=46.9, \mathrm{MSE}=67240.0, P<0.001)$. The sexby-category interaction was also not significant, $F(1,9)=0.005$, $\mathrm{MSE}=5.62, P<0.94$. From Figure 8, the no interaction appears to be due to the larger category effect for cross vs within only but not for the sex male vs female. This impression was supported by paired samples $t$-tests (two-tailed) used to investigate the category for each sex. There was a significant category effect for the male $(t(9)=4.59, P<0.001)$, and also for the female $(t(9)=6.05, P=0.001)$.

\section{Discussion}

For both male and female Arabic participants, discrimination of pairs of colors from different lexical categories (blue and purple) was faster than pairs from the same lexical categories (different shades of blue or purple). Yet again, the pattern of color categorization has been replicated, but this time, the category effect from right-to-left script readers in a secondary color category boundary has also been shown. All of the research on color $\mathrm{CP}$ before this study was conducted with Roman script or top-to-bottom scripts. But in this experiment, using participants of right-to-left scripts, such as Arabic, the author show essentially the same pattern as the color CP. Thus, these data provide further support for the color CP.

\section{General discussion}

The overall aim of the experiments presented in this paper was to establish the Arabic blue-purple category boundary and in particular to assess whether previous findings of $\mathrm{CP}$ of color can be shown in participants whose reading direction was from right to left as in Arabic in (blue-purple) secondary color category boundary.

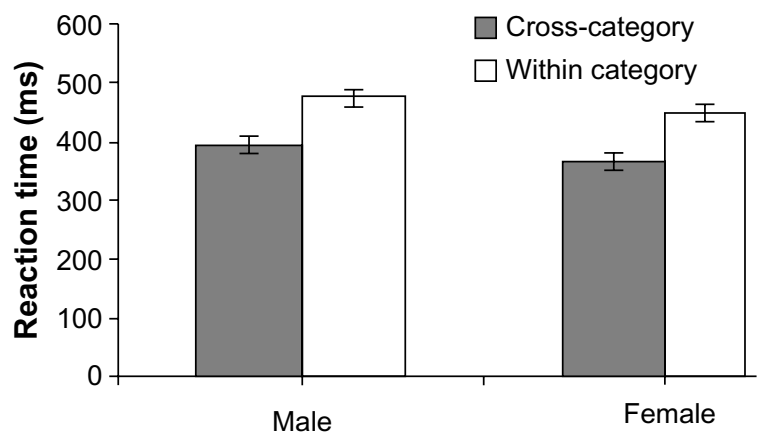

Figure 8 Mean response times $( \pm \mathrm{SE})$ with SE bars for correct trials for male and female participants for identification of the within/cross-category of chromatic target among distractors.

Abbreviation: SE, standard error. 
The previous studies that found CP of $\operatorname{color}^{24,29,38-46}$ have all tested participants who read left-to-right scripts (or topto-bottom scripts), but participants who read from right to left in secondary color category had not been tested. Reading direction affects the pattern of lateralization on a range of perceptual tasks, ${ }^{46,53,54,56-58}$ so it was plausible that reading direction could affect the CP of color. This hypothesis was tested by testing the CP of color of blue-purple in Arabic participants as (secondary color category). Experiments 1 and 2 identified the location of the Arabic blue-purple category boundary. The findings suggested that the blue-purple category boundary for an Arabic speaker is around 10PB corresponding to the English blue-purple category boundary reported by Franklin and Davies. ${ }^{61}$ Then, the CP of color was investigated in Experiment 3 for Arabic speakers - two groups of participants who differ in their sex took part in this experiment. Experiment 3 used a grid search task with an RT measure, while it was found that both groups had shown the CP of color. This confirms the robust nature of the color CP.

In summary, the present results of this study revealed color CP. The CP of color appears not to be affected by reading direction, and the effect is found for participants who read from right to left in primary color category as in a study by Al-rasheed ${ }^{11}$ and in secondary color category as in the current finding and on a range of different visual search tasks.

\section{Disclosure}

The author reports no conflicts of interest in this work.

\section{References}

1. Mollon JD. Color vision: opsins and options. Proc Natl Acad Sci USA. 1999;96:4743-4745.

2. Heider ER. Universals in color naming and memory. $J$ Exp Psychol. 1972;93(1):10-20.

3. Kay P, Berlin B, Merrifield W. Biocultural implications of systems of color naming. J Linguist Anthropol. 1991;1:12-25.

4. MacLaury RE. Color-category evolution and Shuswap yellow-withpurple. Am Anthropol. 1987;89:107-124.

5. Newhall S, Nickerson D, Judd D. Final report of the O.S.A. subcommittee on spacing of the Munsell colors. J Opt Soc Am. 1943;33:385-418.

6. CIE. Commission internationale de l'Eclairage proceedings. Cambridge: Cambridge University Press; 1932.

7. Al-rasheed A, Al-Sharif H, Thabit M, Al-Mohimeed N, Davies I. Basic colour terms of Arabic. In: Biggam, Carole P, Carole A, et al., editors. New Directions in Colour Studies. Amsterdam: John Benjamins Publishing Company; 2011:2.

8. Al-rasheed A, Al-Mohimeed N, Davies I. Berlin and Kay's theory of color universals and linguistic relativity. The case of Arabic. J Mod Educ Rev. 2013;2(2):45-62.

9. Al-rasheed A. Further evidence for Arabic basic colour categories. Psychology. 2014;5:1714-1729.

10. Berlin B, Kay P. Basic Color Terms: Their Universality and Evolution. Berkeley, Los Angeles: University of California Press; 1969.
11. Al-rasheed A. Categorical effects in the perception of colour: behavioral evidence in hue search method. Psychol Res. 2014;4(8):623-634.

12. Cohen J. Color ontology and its significance. In: Symons J, Calvo P, editors. The Routledge Companion to the Philosophy of Psychology. (Chap. 36). Taylor and Francis Ltd; 2009.

13. Newton I. Opticks: Or a Treatise on the Reflections, Refractions and Colours of Light. 4th ed. New York: Dover Publications; 1730/1952.

14. Maxwell JC. Experiments on Colour as Perceived by the Eye, with Remarks on Colour Blindness. Transactions of the Royal Society of Edinburgh; 1855):XXL, Part2.

15. Gladstone WE. Studies on Homer and the Homeric Age. London: Oxford University Press; 1858:III, 457-499.

16. Geiger L. Contributions to the History of the Development of the Human Race. London: Tubner and Company; 1880. [Translated by D. Asher].

17. Rivers C. Reports of the Cambridge Anthropological Expedition to the Torres Straits, 2. Cambridge: Cambridge University Press; 1901.

18. Whorf BL. Language Thought and Reality. Cambridge, MA: MIT Press; 1956.

19. Magnus H. Die geschichtliche Entwickelung des Farbensinne. Leipzig: Von Veit; 1877.

20. Woodworth RS. The puzzle of color vocabularies. Psychol Bull. 1910;7: 325-334.

21. Ray VF. Techniques and problems in the study of human color perception. Southwest J Anthropol. 1952;8(3):251-259.

22. Kay P, McDaniel C. The linguistic significance of the meanings of basic color terms. Language. 1978;54:610-646.

23. Saunders BAC, van Brakel J. Ate there non-trivial constraints on color categorization? Behav Brain Sci. 1997;20:167-232.

24. Roberson D, Davies IRL, Davidoff J. Color categories are not universal: replications and new evidence from a stone-age culture. J Exp Psychol Gen. 2000;129:369-398.

25. Brown R, Lenneberg EH. A study on language and cognition. J Abnorm Soc Psychol. 1954;49:454-462.

26. Heider ER, Olivier CC. The structure of the color space in naming and memory for two languages. Cogn Psychol. 1972;3:337-354.

27. Rosch, E. Natural categories. Cognitive Psychology. 1973;4:328-350.

28. Davies IRL, Corbett GG. A cross-cultural study of colour grouping: evidence for weak linguistic realtivity. Br J Psychol. 1997;88:493-517.

29. Bornstein MH, Korda N. Discrimination and matching within and between hues measured by reaction times: some implications for categorical perception and levels of information processing. Psychol Res. 1984;46:207-222.

30. Mollon JD. "Cherries among the leaves": the evolutionary origins of colour vision. In: Davis S, editor. Colour Perception: Philosophical, Psychological, Artistic, and Computational Perspective. Oxford: Oxford University Press; 2000:10-30.

31. Gegenfurtner KR, Kiper DC. Color vision. Annu Rev Neurosci. 2003;26:181-206.

32. Conway BR. Color vision, cones, and color-coding in the cortex. Neuroscientist. 2009;15:274-290.

33. Krauskopf J, Williams DR, Heeley DW. The cardinal directions of color space. Vision Res. 1982;22(9):1123-1131.

34. De Valois RL, Jacobs GH. Spectral sensitivity in monkey. Science. 1968;162:533-540.

35. Hering ER. Outlines of a Theory of the Light Sense. Cambridge, MA: Harvard University Press; 1920/1964. [Translated by L M Hurvich and D Jameson].

36. De Valois RL, De Valois KK. A multi-stage color model. Vision Res. 1993;33(8):1053-1065.

37. Harnad S. Categorical Perception: The Groundwork of Cognition. New York: Cambridge University Press; 1987.

38. Uchikawa K, Shonida H. Influence of basic color categories on color memory discrimination. Color Res Appl. 1996;21:430-439.

39. Roberson D, Davidoff J, Braisby N. Similarity and categorisation: neuropsychological evidence for a dissociation in explicit tasks. Cognition. 1999;71:1-42. 
40. Roberson D, Davidoff J. The categorical perception of colors and facial expressions. The effect of verbal interference. Mem Cogn. 2000;28: 977-986.

41. Pilling M, Wiggett A, Özgen E, Davies IRL. Is color "categorical perception" really perceptual? Mem Cogn. 2003;31:538-551.

42. Boynton RM, Fargo L, Olson CX, Smallman HS. Category effect in color memory. Color Res Appl. 1989;14:229-234.

43. Laws G, Davies I, Andrews C. Linguistic structure and non-linguistic cognition: English and Russian blues compared. Lang Cogn Process. 1995;10:59-94.

44. Franklin A, Pilling M, Davies IRL. The nature color categorisation: evidence from eye-movements on a target detection task. J Exp Child Psychol. 2005;91:227-248.

45. Daoutis CA, Franklin A, Riddet A, Clifford A, Davies IRL. Categorical effects in children's colour search: a cross-linguistic comparison. $\mathrm{Br} J$ Dev Psychol. 2006;24(2):373-400.

46. Daoutis CA, Pilling M, Davies IRL. Categorical effects in visual search of colour. Vis cogn. 2006;14:229-234.

47. Gilbert AL, Regier T, Kay P, Ivry RB. Whorfian hypothesis supported in the right visual field but not the left. Proc Natl Acad Sci USA. 2006;103:489-494.

48. Drivonikou GV, Kay T, Regier RB, Ivry AL, Franklin A, Davies IRL. Further evidence that Whorfian effects are stronger in the right visual field than the left. Proc Natl Acad Sci US A. 2007;104: 1097-1102.

49. Roberson D, Park H, Hanley RJ. Categorical perception of colour in the left and right visual field is verbally mediated: evidence from Korean. Cognition. 2008;107(2):752-762.

50. Siok WT, Kay P, Wang WSY, et al. Language regions of brain are operative in color perception. Proc Natl Acad Sci U S A. 2009;106(20): 8140-8145.
51. Liu Q, Li H, Campos JL, et al. The N2pc component in ERP and lateralization effect of language on color perception. Neurosci Lett. 2009;454: $58-61$.

52. Al-rasheed A, Franklin A, Drivonikou G, Davies I. Left hemisphere lateralization of categorical color perception among roman and Arabic script readers. Psychology. 2014;5:255-270.

53. Eviatar Z. Reading direction and attention: effects on lateralized ignoring. Brian Cogn. 1995;29:137-150.

54. Eviatar Z. Language experience and right hemisphere task: the effects of scanning habits and multilingualism. Brian Cogn. 1997;58:157-173.

55. Farid M, Grainger J. How initial fixation position influences visual word recognition: a comparison of French and Arabic. Brian Lang. 1996;53: $351-368$.

56. Prunet JF, Beland R, Idrissi A. The mental representation of semitic word. Linguist Inq. 2000;31:609-648.

57. Berent I. Identity avoidance in the Hebrew lexicon: implications for symbolic accounts of word formation. Brian Lang. 2002;81:326-341.

58. Schwalm ND, Eviatar Z, Golan Y, Blumenfeld Y. The effect of reading direction habit on numerical processing. In: Proceeding of the Human Factors and Ergonomics Society, 47th, Annual Meeting; 2003:1649-1653.

59. Han S, NorthoffG. Culture-sensitive neural substrates of human cognition: a transcultural neuroimaging approach. Nat Rev Neurosci. 2008;9: 646-654.

60. Fletcher R. The City University Colour Vision Test. Windsor, Berks: Keeler Ltd; 1980.

61. Franklin A, Davies IR. New evidence for infant colour categories. $\mathrm{Br}$ J Dev Psychol. 2004;22:349-377. 


\section{Supplementary materials}

\section{The Munsell color-order system}

The Munsell color-order system developed by an American artist Albert H Munsell in 1905 was designed to provide an orderly system for accurately identifying every perceptible color. The system specifies color in terms of three attributes: hue, value, and chroma (Figure S1).

The system has five principal hues on the horizontal plane - red (R), yellow $(\mathrm{Y})$, purple $(\mathrm{G})$, blue $(\mathrm{B})$, and purple (P) - and five intermediate hues - yellow-red (YR), purpleyellow (GY), blue-purple (BG), blue-purple (PB), and redpurple (RP) - making ten hues in all. The vertical plane gives the value, which indicates the lightness of color, and distinguishes light colors from dark ones. The value scale ranges from 0 for pure black to 10 for pure white with different shades of gray between them. The horizontal plane represents the chroma, which indicates the saturation of colors. The Munsell color-order system is standardized so that each of the three Munsell dimensions is intended to be perceptually uniform. ${ }^{5}$

\section{The Commission Internationale de l'Eclairage}

In 1931, the Commission Internationale de 1'Eclairage (International Commission on Illumination) (CIE) produced the well-known color space that represents all possible colors in a chromaticity diagram. This model has been developed in several versions. One of them is the 1976 uniform chromaticity CIE $\left(u^{\prime}, v^{\prime}\right)$ that was used in the experiments of the current study. This version is designed to be perceptually uniform. A given change in value corresponds nearly to the same perceptual difference over any part of the space. Table S1 shows the color-aid codes and CIE coordinates for the stimuli used for the color naming in Experiment 2.

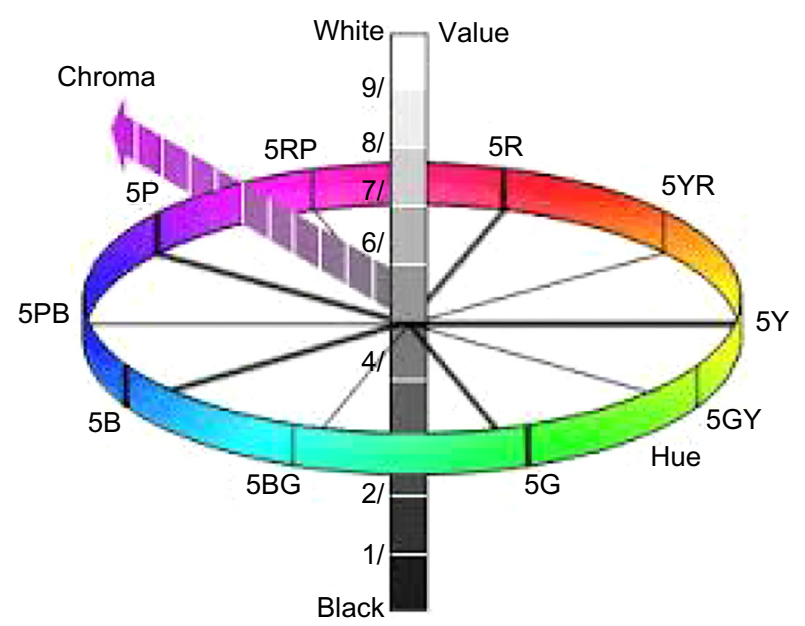

Figure SI Schematic representation of Munsell color space.
Table SI Color-aid codes and CIE coordinates stimuli used for the color naming

\begin{tabular}{|c|c|c|c|c|c|c|}
\hline \multirow{2}{*}{$\begin{array}{l}\text { Color-aid } \\
\text { code }\end{array}$} & \multicolumn{6}{|c|}{ CIE coordinates } \\
\hline & $Y$ & $x$ & $y$ & $L^{*}$ & $u^{\prime}$ & $v^{\prime}$ \\
\hline \multicolumn{7}{|l|}{$Y$} \\
\hline HUE & 64.77 & 0.47 & 0.48 & 91.49 & 0.24 & 0.55 \\
\hline S2 & 16.99 & $0.4 I$ & 0.44 & 52.81 & 0.22 & 0.53 \\
\hline \multicolumn{7}{|l|}{ YOY } \\
\hline HUE & 47.48 & 0.50 & 0.43 & 80.92 & 0.28 & 0.54 \\
\hline $\mathrm{T} 4$ & 55.63 & 0.45 & 0.41 & 86.18 & 0.26 & 0.53 \\
\hline S2 & 22.08 & 0.36 & 0.38 & 59.09 & 0.21 & 0.50 \\
\hline \multicolumn{7}{|l|}{ YO } \\
\hline HUE & 39.52 & 0.51 & $0.4 I$ & 75.17 & 0.30 & 0.53 \\
\hline $\mathrm{T} 3$ & 47.02 & 0.48 & $0.4 \mathrm{I}$ & 80.61 & 0.28 & 0.53 \\
\hline S3 & 10.72 & 0.36 & 0.41 & 43.02 & 0.20 & 0.51 \\
\hline \multicolumn{7}{|l|}{ OYO } \\
\hline HUE & 26.51 & 0.54 & 0.37 & 63.81 & 0.34 & 0.52 \\
\hline \multicolumn{7}{|l|}{$\mathrm{O}$} \\
\hline HUE & 25.00 & 0.54 & 0.37 & 62.26 & 0.34 & 0.52 \\
\hline SI & 14.34 & 0.50 & 0.37 & 49.03 & 0.31 & 0.52 \\
\hline S3 & 09.15 & 0.42 & 0.36 & 39.98 & 0.26 & 0.50 \\
\hline \multicolumn{7}{|l|}{ ORO } \\
\hline HUE & 18.87 & 0.57 & 0.34 & 55.26 & 0.38 & 0.52 \\
\hline T3 & 36.88 & 0.46 & 0.35 & 73.09 & 0.29 & 0.50 \\
\hline S3 & 26.51 & 0.33 & 0.32 & 63.81 & 0.21 & 0.47 \\
\hline \multicolumn{7}{|l|}{ RO } \\
\hline HUE & 16.22 & 0.58 & 0.33 & 51.75 & 0.40 & 0.51 \\
\hline $\mathrm{T} 3$ & 32.66 & 0.45 & 0.32 & 69.56 & 0.30 & 0.48 \\
\hline S3 & 04.19 & 0.37 & 0.34 & 27.15 & 0.23 & 0.48 \\
\hline \multicolumn{7}{|l|}{ ROR } \\
\hline HUE & 15.23 & 0.53 & 0.31 & 50.35 & 0.37 & 0.49 \\
\hline $\mathrm{T} 3$ & 29.82 & 0.42 & 0.30 & 67.00 & 0.29 & 0.47 \\
\hline S3 & 20.71 & 0.34 & 0.28 & 57.50 & 0.24 & 0.44 \\
\hline \multicolumn{7}{|l|}{$\mathrm{R}$} \\
\hline HUE & 11.71 & 0.50 & 0.29 & 44.78 & 0.36 & 0.48 \\
\hline $\mathrm{T} 4$ & 24.34 & 0.40 & 0.27 & 61.57 & 0.29 & 0.45 \\
\hline S3 & 04.81 & 0.33 & 0.30 & 29.18 & 0.22 & 0.45 \\
\hline \multicolumn{7}{|l|}{ RVR } \\
\hline HUE & 09.11 & 0.42 & 0.24 & 39.90 & 0.33 & 0.43 \\
\hline SI & 12.79 & 0.35 & 0.25 & 46.60 & 0.26 & 0.42 \\
\hline S3 & 28.43 & 0.36 & 0.28 & 65.69 & 0.26 & 0.45 \\
\hline \multicolumn{7}{|l|}{ RV } \\
\hline HUE & 06.97 & 0.33 & 0.19 & 35.13 & 0.29 & 0.37 \\
\hline $\mathrm{T} 2$ & $|4.5|$ & 0.31 & 0.19 & 49.28 & 0.27 & 0.37 \\
\hline \multicolumn{7}{|l|}{ VRV } \\
\hline HUE & 06.7I & 0.30 & 0.19 & 34.48 & 0.26 & 0.37 \\
\hline S3 & 08.42 & 0.36 & 0.28 & 65.68 & 0.26 & 0.45 \\
\hline \multicolumn{7}{|l|}{$V_{-}$} \\
\hline HUE & 04.67 & 0.26 & 0.17 & 28.74 & 0.23 & 0.34 \\
\hline \multicolumn{7}{|l|}{ VBV } \\
\hline HUE & 04.13 & 0.24 & 0.17 & 26.94 & 0.21 & 0.34 \\
\hline $\mathrm{T} 4$ & 19.05 & 0.25 & 0.20 & 55.49 & 0.20 & 0.37 \\
\hline \multicolumn{7}{|l|}{ BV } \\
\hline HUE & 04.21 & 0.22 & 0.19 & 27.22 & 0.18 & 0.35 \\
\hline S2 & 07.88 & 0.25 & 0.26 & 37.26 & 0.18 & 0.42 \\
\hline \multicolumn{7}{|l|}{ BVB } \\
\hline HUE & 04.80 & 0.19 & 0.13 & 29.15 & 0.18 & 0.28 \\
\hline S3 & 26.65 & 0.26 & 0.23 & 63.95 & 0.20 & 0.40 \\
\hline
\end{tabular}


Table SI (Continued)

\begin{tabular}{|c|c|c|c|c|c|c|}
\hline \multirow{2}{*}{$\begin{array}{l}\text { Color-aid } \\
\text { code }\end{array}$} & \multicolumn{6}{|c|}{ CIE coordinates } \\
\hline & $Y$ & $x$ & $y$ & $L^{*}$ & $u^{\prime}$ & $v^{\prime}$ \\
\hline \multicolumn{7}{|l|}{$B$} \\
\hline HUE & 09.51 & 0.18 & 0.16 & 40.71 & 0.16 & 0.32 \\
\hline $\mathrm{TI}$ & 19.02 & 0.20 & 0.19 & 55.45 & 0.16 & 0.35 \\
\hline \multicolumn{7}{|l|}{ BGB } \\
\hline HUE & 09.62 & 0.19 & 0.19 & 40.93 & 0.16 & 0.35 \\
\hline T3 & 23.08 & 0.20 & 0.23 & 60.21 & 0.15 & 0.39 \\
\hline \multicolumn{7}{|l|}{ BG } \\
\hline HUE & 08.93 & 0.20 & 0.25 & 39.53 & 0.14 & 0.40 \\
\hline $\mathrm{TI}$ & 16.57 & 0.19 & 0.25 & 52.24 & 0.14 & 0.40 \\
\hline $\mathrm{S} 2$ & 07.42 & 0.21 & 0.26 & 36.21 & 0.15 & 0.41 \\
\hline \multicolumn{7}{|l|}{ GBG } \\
\hline HUE & 10.69 & 0.23 & 0.37 & 42.96 & 0.13 & 0.48 \\
\hline $\mathrm{S} 2$ & 20.79 & 0.20 & 0.25 & 57.60 & 0.14 & 0.40 \\
\hline \multicolumn{7}{|l|}{ G } \\
\hline HUE & 11.99 & 0.24 & 0.42 & 45.26 & 0.13 & 0.50 \\
\hline S3 & 06.10 & 0.26 & 0.33 & 32.91 & 0.16 & 0.46 \\
\hline \multicolumn{7}{|l|}{ GYG } \\
\hline HUE & 12.89 & 0.25 & 0.44 & 46.76 & 0.13 & 0.51 \\
\hline $\mathrm{T} 4$ & 31.14 & 0.26 & 0.41 & 68.21 & 0.14 & 0.50 \\
\hline SI & 15.59 & 0.26 & 0.31 & 50.86 & 0.17 & 0.45 \\
\hline \multicolumn{7}{|l|}{ YG } \\
\hline HUE & 14.66 & 0.28 & 0.48 & 49.51 & 0.14 & 0.53 \\
\hline S3 & 05.78 & 0.30 & 0.34 & 32.04 & 0.19 & 0.47 \\
\hline \multicolumn{7}{|l|}{ YGY } \\
\hline HUE & 18.92 & 0.30 & $0.5 I$ & 55.32 & 0.14 & 0.54 \\
\hline \multicolumn{7}{|l|}{ YGY } \\
\hline S3 & 35.87 & 0.35 & 0.43 & 72.27 & 0.19 & 0.52 \\
\hline ROSERED & 17.63 & $0.4 I$ & 0.24 & 53.66 & 0.32 & 0.43 \\
\hline SIENNA & $|3.3|$ & 0.44 & 0.36 & 47.43 & 0.27 & 0.50 \\
\hline WHITE & 81.40 & 0.32 & 0.33 & 100.00 & 0.20 & 0.47 \\
\hline GRAY I & 47.55 & 0.32 & 0.33 & 80.97 & 0.20 & 0.47 \\
\hline GRAY 2 & 30.59 & 0.32 & 0.33 & 67.71 & 0.20 & 0.47 \\
\hline GRAY 4 & 18.88 & 0.31 & 0.31 & 55.27 & 0.20 & 0.46 \\
\hline GRAY 6 & 11.20 & 0.31 & 0.31 & 43.89 & 0.20 & 0.46 \\
\hline GRAY 8 & 04.53 & 0.31 & 0.32 & 28.89 & 0.20 & 0.46 \\
\hline BLACK & 03.59 & 0.34 & 0.33 & 24.98 & 0.22 & 0.47 \\
\hline
\end{tabular}

Abbreviation: CIE, Commission Internationale de l'Eclairage.

\section{Publish your work in this journal}

Psychology Research and Behavior Management is an international, peerreviewed, open access journal focusing on the science of psychology and its application in behavior management to develop improved outcomes in the clinical, educational, sports and business arenas. Specific topics covered include: Neuroscience, memory \& decision making; Behavior modification \& management; Clinical applications; Business \& sports performance management; Social and developmental studies; Animal studies. The manuscript management system is completely online and includes a quick and fair peer-review system. Visit http://www.dovepress. com/testimonials.php to read real quotes from published authors. 